03,04

\title{
Электронная структура и нелинейная диэлектрическая восприимчивость $\gamma$-фазы оксида теллура
}

\author{
(C) Е.М. Рогинский ${ }^{1}$, М.Б. Смирнов ${ }^{2}$ \\ ${ }^{1}$ Физико-технический институт им. А.Ф. Иофрфе РАН, \\ Санкт-Петербург, Россия \\ ${ }^{2}$ Санкт-Петербургский государственный университет, \\ Санкт-Петербург, Россия \\ E-mail: e.roginski@@mail.ioffe.ru
}

Поступила в Редакцию 25 ноября 2019 г.

В окончательной редакции 25 ноября 2019 г.

Принята к публикации 28 ноября 2019 г.

Теоретически с использованием неэмпирических квантово-механических расчетов изучены структурные, электронные и нелинейные оптические свойства кристалла $\gamma-\mathrm{TeO}_{2}$. Учет локализации электронов на $5 d$-орбитали проведен с использованием поправок Хаббарда к функционалу плотности (приближение $\mathrm{LDA}+U)$. Использование такого подхода позволило достаточно точно воспроизвести экспериментальные структурные параметры. Электронная структура изучена с использованием квазичастичного приближения $G_{0} W_{0}$, зарекомендовавшим себя как один из наиболее точных методов расчета зонной структуры. Установлено, что кристалл $\gamma-\mathrm{TeO}_{2}$ представляет собой широкозонный полупроводник с непрямым оптическим переходом. При помощи максимально локализованных функций Ванье выполнен анализ химической связи в этом оксиде и показано, что валентные электроны атомов кислорода находятся в sp3 гибридизации, а валентность атомов теллура равна четырем.

Ключевые слова: нелинейная оптика, оксиды теллура, ab initio расчеты.

DOI: $10.21883 /$ FTT.2020.04.49117.641

\section{1. Введение}

Среди большого разнообразия кристаллических и стеклообразных твердых тел оксиды теллура выделяются уникальными нелинейных оптическими свойствами. Стекла на основе диоксида теллура имеют нелинейную диэлектрическую восприимчивость в 50 раз превосходящую аналогичную характеристику силикатных стекол [1]. Одна из кристаллических модификаций диоксида теллура - парателлурит $\left(\alpha-\mathrm{TeO}_{2}\right)$ - широко применяется в разнообразных устройствах ввиду выдающихся акусто-оптических, диэлектрических и электрооптических характеристик [2]. Есть основания предполагать, что другие модификации $\mathrm{TeO}_{2}$ могут иметь столь же выдающиеся (и даже превосходящие) диэлектрические свойства. Особый интерес привлекает кристаллическая структура $\gamma-\mathrm{TeO}_{2}$ [3], поскольку она является промежуточной фазой между стеклообразным $\mathrm{TeO}_{2}$ и парателлуритом. Ввиду метастабильности этой фазы получение экспериментальных оценок ее диэлектрических восприимчивостей затруднительно. Поэтому расчет этих характеристик с использованием высокоточных квантово-механических методов является весьма актуальной задачей.

Однако выбор схемы расчета, позволяющей достаточно точно оценить диэлектрические восприимчивости (как линейные, так и не линейные) не прост. Стандартное LDA-приближение хорошо предсказывает геометрию структуры, в которой важную роль играют межмолекулярные взаимодействия, однако сильно переоценивает обменно-корреляционное слагаемое в полной энергии [4], что приводит к известной проблеме недооценки ширины запрещенной зоны, и, как следствие, к ошибкам определения диэлектрических свойств изучаемых структур. Наиболее точный расчет полной энергии можно получить в рамках приближения случайных фаз [5], в котором обменно-корреляционный член полной энергии $E_{x c}^{L D A}$ заменяется квазичастичным обменным членом $E_{x}^{Q P}$ и корреляционным слагаемым $E_{c}^{R P A}$. Однако такие расчеты довольно ресурсоемкие, поэтому имеют ограниченное применение к высокосимметричным структурам с небольшим числом атомов в примитивной ячейке.

Кроме указанного выше подхода широкую известность получил метод квазичастичного приближения $G W$, моделирующего собственную энергию квазичастиц произведением функции Грина и динамически экранированного кулоновского потенциала. Формально это приближение представляет собой первый член разложения собственно энергетической части в ряд по степеням экранированного кулоновского потенциала. Данный метод зарекомендовал себя как один из самых точных подходов для предсказания экспериментальной электронной зонной структуры [6].

В настоящей работе выполнен сравнительный анализ озвученных выше подходов для кристаллов $\gamma-\mathrm{TeO}_{2}$ с целью выявить наиболее надежный из них для того, чтобы предсказать электронную структуру и диэлектрические свойства кристалла. 


\section{2. Техника вычислений}

Расчеты из первых принципов проводились в рамках метода функционала плотности с помощью программы ABINIT $[7,8]$. В расчетах использовались функционалы, обменно-кореляционная часть которых описывалась в приближении локальной плотности LDA [9]. С целью более корректного учета локализации $5 d$-электронов на атомах теллура в расчетах учитывались поправки Хаббарда к функционалу плотности (схема LDA $+U$ ) [10]. Значение параметра $U$ было позаимствовано из статьи [11] для $\alpha$-фазы парателлурита ввиду близости структурного строения. Оптимизированные нелокальные (с двумя проекторами нелокальной части) сохраняющие норму псевдопотенциалы для атомов Те и О, использовались в настоящих расчетах [12]. В расчетах в качестве валентных считались электроны на орбиталях $2 s 2 p$ для атома О и $5 d 6 s 6 p$ для атома Те. Энергия обрезания при расчете электронной структуры составляла $40 \mathrm{a} . \mathrm{u}$. Для интегрирования по зоне Бриллюэна (ЗБ) использовались сетки Монхорста-Пака [13] размером $6 \times 4 \times 6$. В расчетах проводилась полная релаксация как положений атомов, так и параметров решетки. Релаксация проводилась до тех пор, пока силы, действующие на атомы, не становились менее $2 \cdot 10^{-5} \mathrm{Ha} / \mathrm{Bohr}$ при самосогласованном расчете полной энергии с точностью лучше $10^{-8} \mathrm{Ha}$, а отклонение от нулевого давления не превышало $0.1 \mathrm{kbar}$. Зависимость поляризации от внешнего электрического поля рассчитывалась методом фазы Берри [14]. Квадратичная нелинейная восприимчивость $\chi^{(2)}$ вычислялась путем аппроксимации зависимости поляризации от напряженности электрического поля $P(\mathscr{E})$ квадратичным многочленом. Поляризация рассчитывалась для напряженности электрического поля $\mathscr{E}$ в диапазоне от 0 до 0.001 a.u. с шагом 0.0001 a.u. $\left(1 \mathrm{a} . \mathrm{u} .=5.14 \cdot 10^{7} \mathrm{~V} / \mathrm{m}\right)$. Условием сходимости при определении значения $\chi^{(2)}$ было выбрано условие изменения величины $\chi^{(2)}$ менее чем на $1 \%$ при увеличении на единицу числа точек $P_{n}\left(\mathscr{E}_{n}\right)$, аппроксимируемых квадратичным многочленом.

Электронная структура и диэлектрические проницаемости $\gamma-\mathrm{TeO}_{2}$ рассчитывались в рамках квазичастичного приближения $G_{0} W_{0}[15]$. В качестве входных данных использовались волновые функции и собственные значения энергий, найденные в приближении LDA $+U$. Диэлектрическая матрица $\varepsilon_{G G^{\prime}}(q, \omega)$ вычислялась на сетке из 48 точек в ЗБ из матрицы поляризуемости $P_{G G^{\prime}}(q, \omega)$, рассчитанной в приближении RPA для 56 заполненных и 250 незаполненных зон. Динамическое экранирование описывалось однополюсной плазмонной моделью Годби-Нидса. В расчетах использовались фурьекомпоненты волновых функций с максимальной кинетической энергией 35 a.u. Поправки к энергиям, найденным в приближении $\mathrm{LDA}+U$, рассчитывались как диагональные матричные элементы оператора $\left[\Sigma-E_{x c}\right]$, где $\Sigma=G W$ - оператор собственной энергии, $E_{x c}-$ оператор обменно-корреляционной энергии, $G-$ функция Грина, а $W=\left[\varepsilon^{-1} v\right]$ - оператор экранированного кулоновского взаимодействия. При расчете $\Sigma$ использовались компоненты волновой функции с кинетической энергией до $35 \mathrm{a}$.u. как для обменной, так и для корреляционной частей.

\section{3. Структурные свойства}

$\gamma$-фаза диоксида теллура впервые была синтезирована в 2000 г. [3] путем медленного нагревания чистого стеклообразного $\mathrm{TeO}_{2}$ до температуры $390^{\circ} \mathrm{C}$ с последующим отжигом в течении $24 \mathrm{~h}$. Это метастабильная фаза и при нагревании испытывает реконструктивный необратимый фазовый переход в стабильную $\alpha$-фазу. Основной структурной единицей тетрагонального полиморфа оксида теллура $\alpha-\mathrm{TeO}_{2}$ (пространственная симметрия $\left.P 4{ }_{1} 2{ }_{1}\right)$ является дисфеноид, с двумя длинами связи, а именно экваториальной $\left(\mathrm{Te}^{-} \mathrm{O}_{e q}\right)$ и аксиальной $\left(\mathrm{Te}-\mathrm{O}_{a x}\right)$. Пространственная группа $\gamma-\mathrm{TeO}_{2} \quad\left(P 22_{1} 2_{1} 2_{1}\right)$ принадлежит к классу энантиоморфных групп, в ней есть только одна общая позиция Уайкофа и эта группа одна из самых распространенных для биомолекулярных кристаллов [16], у нее нет выделенного направления и она позволяет (за счет наличия 3 винтовых осей) плотно упаковывать молекулы в элементарной ячейке. Из-за того, что симметрия $\gamma$-фазы ниже $\left(P 2{ }_{1} 2{ }_{1} 2_{1}\right)$, все 4 связи атомов Те с соседними атомами О отличаются по величине, что приводит к искаженной форме дисфеноида. Однако структура кристалла $\gamma-\mathrm{TeO}_{2}$, так же как и в случае с $\alpha-\mathrm{TeO}_{2}$, состоит из трехмерной сетки, образованной из формульных единиц $\mathrm{TeO}_{4}$ с объединенными вершинами. Протекание реконструктивных фазовых переходов может быть рассмотрено в рамках теории представления двух фаз в одной из общих подгрупп [17]. В данном случае одной из таких подгрупп является группа орторомбического полиморфа $P 2_{1} 2_{1} 2_{1}$. Элементарная ячейка $\gamma-\mathrm{TeO}_{2}$ приведена на рис. 1 , а

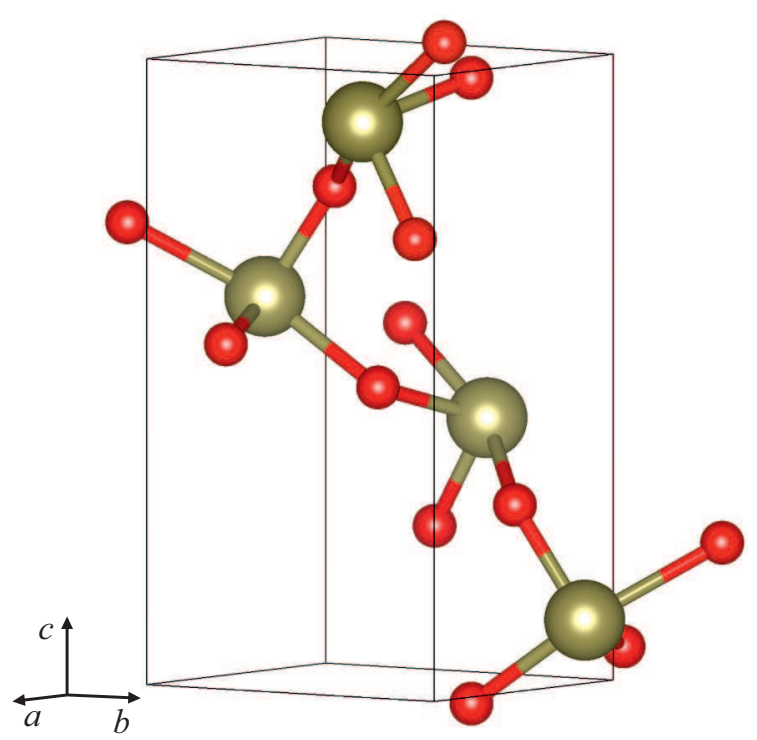

Рис. 1. Элементарная ячейка кристалла $\gamma-\mathrm{TeO}_{2}$. Большие шарики - атомы теллура, маленькие шарики - атомы кислорода. 
Параметры решетки, координаты атомов и основные межатомные расстояния в кристалле $\gamma-\mathrm{TeO}_{2}$. (Атомы Те и О занимают позиции Уайкофа $4 a$, следовательно имеется 3 свободных параметра $(u, v, w))$

\begin{tabular}{c|c|c|c|c}
\hline Параметр & Эксперимент & Эксперимент $^{2}$ & PBE $^{3}$ & $\mathrm{LDA}+\mathrm{U}$ \\
\hline \multicolumn{5}{|c}{ Параметры решетки } \\
$a, \AA$ & 4.8080 & 4.75001 & 5.176 & 8.5001 \\
$b, \AA$ & 7.612 & 8.50074 & 8.797 & 4.7501 \\
$c, \AA$ & 4.8080 & 4.22187 & 4.467 & 4.2219 \\
\multicolumn{5}{|c}{ Позиции атомов, отн. ед. } \\
$\mathrm{T} e(u)$ & 0.7232 & 0.9696 & 0.9581 & 0.9805 \\
$\mathrm{~T} e(v)$ & 0.4924 & 0.1016 & 0.1032 & 0.09947 \\
$\mathrm{Te}(w)$ & 0.5268 & 0.1358 & 0.1184 & 0.12850 \\
$\mathrm{O}_{1}(u)$ & 0.7424 & 0.7593 & 0.7641 & 0.7681 \\
$\mathrm{O}_{1}(v)$ & 0.3112 & 0.2812 & 0.2851 & 0.2795 \\
$\mathrm{O}_{1}(w)$ & 0.1114 & 0.1734 & 0.1645 & 0.1772 \\
$\mathrm{O}_{2}(u)$ & 0.8614 & 0.8553 & 0.8599 & 0.8587 \\
$\mathrm{O}_{2}(v)$ & -0.0612 & 0.0362 & 0.0406 & 0.0400 \\
$\mathrm{O}_{2}(w)$ & 0.9924 & 0.7273 & 0.7131 & 0.7162 \\
& Длины связей Те-О, $\AA$ \\
$d\left(T e-O_{1}\right)$ & 1.879 & 1.859 & 1.900 & 1.841 \\
$d\left(T e-O_{2}\right)$ & 1.879 & 1.949 & 1.960 & 1.911 \\
$d\left(T e-O_{1}^{\prime}\right)$ & 2.121 & 2.019 & 2.119 & 2.034 \\
$d\left(T e-O_{2}^{\prime}\right)$ & 2.121 & 2.198 & 2.252 & 2.178
\end{tabular}

Примечание. ${ }^{1}$ Параметры для $\alpha-\mathrm{TeO}_{2}$ из работы [18] в ромбической установке. ${ }^{2}$ Параметры для $\gamma-\mathrm{TeO}_{2}$ из работы[19]. ${ }^{3}$ Параметры из работы[20].

экспериментальные параметры решетки, позиции атомов и длины связей в сравнении с $\alpha$ - $\mathrm{TeO}_{2}$ (в ромбической установке) построены в таблице.

Структурные параметры представленные в таблице, говорят о хорошем согласии выполненных расчетов в приближении LDA $+U$ с экспериментом. В отличии от метода PBE, который несколько завышает параметры решетки, и как следствие, длины связей, приближение, учитывающее поправки Хаббарда к функционалу плотности, немного недоценивает экспериментальные параметры решетки, но с точностью до 2 процентов воспроизводит длины связей, что говорит о хорошем качестве выбора приближения при описании структурных свойств $\gamma-\mathrm{TeO}_{2}$.

На рис. 2 построены проекции $\alpha$ - и $\gamma$-полиморфов оксида теллура на плоскость $(x, y)$ низкосиметричной структуры. Из этого рисунка и данных приведенных в таблице можно легко видеть, что основным отличием этих полиморфов является существенное увеличение параметра $b$ в $\gamma-\mathrm{TeO}_{2}$ и изменение положения атомов $\mathrm{O}_{1}$ и $\mathrm{O}_{2}$ в ячейке относительно оси $b$. Проекции атомов $\mathrm{O}_{2}$ и $\mathrm{O}_{1}^{\prime}$ сближаются в $\gamma-\mathrm{TeO}_{2}$, что и приводит к исчезновению винтовой оси четвертого порядка в этом кристалле. Тем не менее, как указывалось ранее, оба полиморфа имеют схожую структуру, но не смотря на схожесть, понижение симметрии в $\gamma-\mathrm{TeO}_{2}$ приводит к снятию ограничения на вырождение квадратичных нелинейных свойств.

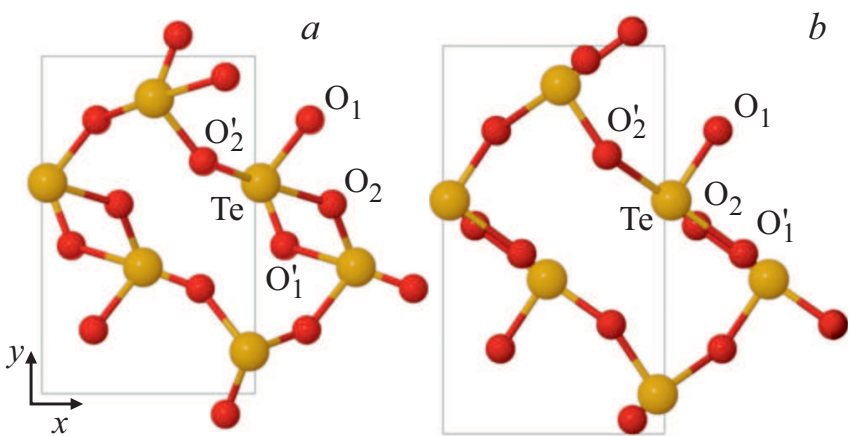

Рис. 2. Проекция элементарных ячеек $\alpha-\mathrm{TeO}_{2}(a)$ и $\gamma-\mathrm{TeO}_{2}(b)$ на плоскость $(x, y)$ в ромбической пространственной группе. Большие шарики - атомы теллура, маленькие шарики атомы кислорода.

\section{4. Электронная структура и анализ химической связи}

Как было показано ранее [11], точность предсказания нелинейных оптических свойств сильно зависит от точности предсказания электронной структуры кристалла. К сожалению, до сих пор ширина запрещенной зоны $\gamma-\mathrm{TeO}_{2}$ не определена экспериментально. Поэтому, в настоящей работе были выполнены прецизионные расчеты в квазичастичном приближении методом $G_{0} W_{0}$, который зарекомендовал себя как один из самых точных подходов при предсказании экспериментальной электронной зонной структуры [6]. Расчеты в квазичастичном приближении чрезвычайно ресурсоемки, поэтому для расчета зонной структуры, полученные на сетке $4 \times 4 \times 4$ точные значения собственной энергии интерполировались с помощью функций Ванье (ВФ), которые строились методом максимальной локализации [21]. Функции Ванье определяются как унитарное преобразование блоховских орбиталей. Однако они определены не однозначно ввиду произвольности фаз блоховских орбиталей. Однако введя критерий минимизации размытия $(\Omega)$ ВФ $w_{n}(r)$, можно устранить эту неоднозначность.

$$
\Omega=\sum_{n=1}^{N}\left(\left\langle w_{n}\left|r^{2}\right| w_{n}\right\rangle-\left\langle w_{n}|r| w_{n}\right\rangle^{2}\right)
$$

Зонная структура кристалла $\gamma$ - $\mathrm{TeO}_{2}$ рассчитанная методом $G_{0} W_{0}$ построена на рис. 3 , координаты высокосимметричных точек ЗБ позаимствованы из работы [22].

Анализируя полученную зонную структуру можно сказать, что максимум валентной зоны находится вблизи центра ЗБ (Г-точка), однако дно зоны проводимости находится в точке, находящейся между точками $X$ и $S$, с координатой $(0.25,0.25,0)$ из чего следует вывод, что, строго говоря, как и в случае с $\alpha$ - $\mathrm{TeO}_{2}, \gamma$-фаза диоксида теллура - это широкозонный полупроводник с непрямым оптическим переходом, при этом ширина прямого перехода который находится в окрестности 


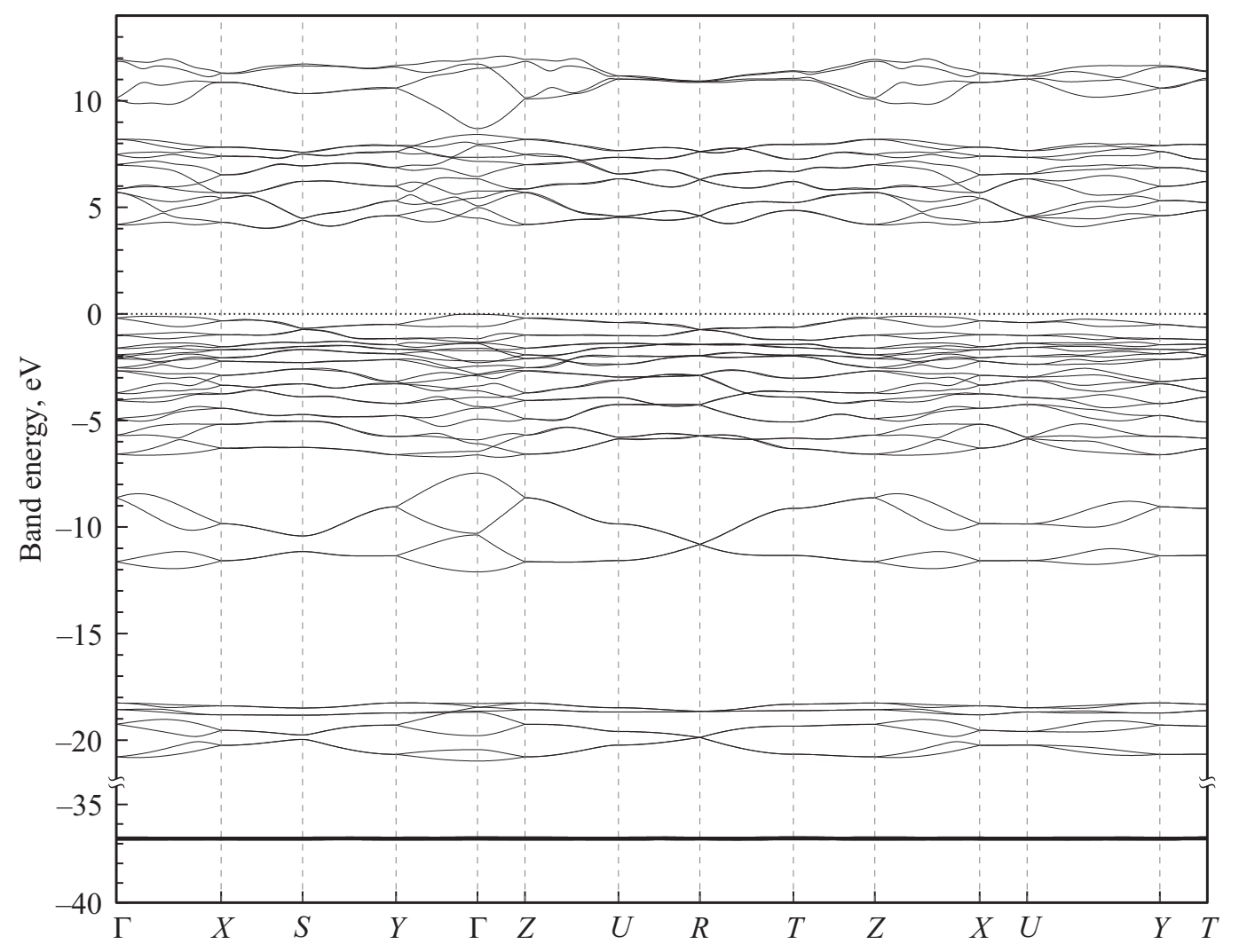

Рис. 3. Электронная зонная структура кристалла $\gamma-\mathrm{TeO}_{2}$ рассчитанная в квазичастичном приближении $G_{0} W_{0}$.
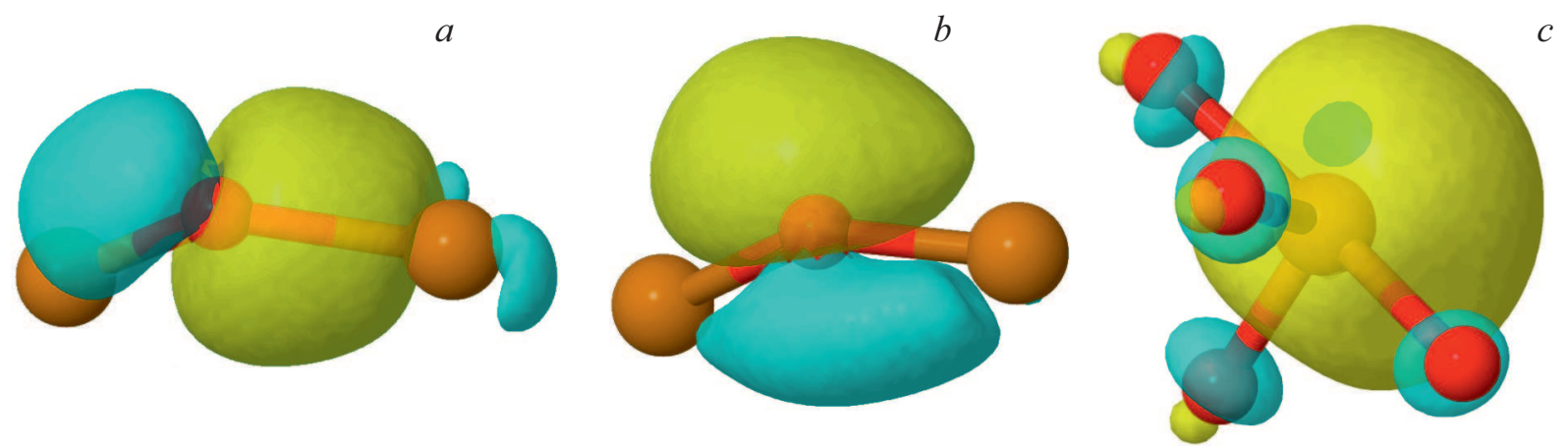

Рис. 4. Пространственная форма максимально локализованных ВФ для кристаллов $\gamma$-ТеО 2 характеризующих орбиталь локализованную на связи Те-О $(a)$, орбиталь неподеленной пары атома $\mathrm{O}(b)$ и орбиталь неподеленной пары атома Те $(c)$.

$Z$-точки ЗБ составляет $\mathrm{E}_{g}^{d}=4.22 \mathrm{eV}$, а величина непрямого перехода равна $\mathrm{E}_{g}^{i}=4.05 \mathrm{eV}$, что является довольно близкими значениями. Сравнивая ширину запрещенной зоны $\gamma-\mathrm{TeO}_{2}$ с шириной $\alpha-\mathrm{TeO}_{2}$, которая составляет $\mathrm{E}_{g}^{d}=3.68 \mathrm{eV}$ [11] можно сделать вывод, что $\gamma$-фаза пропускает свет в более широком диапазоне, что может оказаться полезным при практическом использовании материала в ультрафиолетовом диапазоне.

Для детального анализа зонной структуры необходимо понимание химической связи в кристалле. Наиболее наглядным является метод визуализации пространственной формы максимально локализованных ВФ. На рис. 4 показаны три вида ВФ.
Анализ пространственной формы ВФ показал, что всего имеется три вида орбиталей, а именно по две орбитали на каждый атом О, локализованных на связи $\mathrm{Te}-\mathrm{O}$ с центром на атоме кислорода (рис. 4, $a$ ), по две орбитали на каждый атом кислорода не участвующие в создании химической связи $\mathrm{Te}-\mathrm{O}(\mathrm{BOND})$ с центром на атоме $\mathrm{O}$ и орбиталь неподеленной пары атома $\mathrm{Te}(\mathrm{O}) \mathrm{c}$ центром на атоме $\mathrm{Te}(\mathrm{Te}(\mathrm{LP}))$. Таким образом валентные орбитали атома кислорода имеют $\mathrm{sp}^{3}$ гибридизацию, причем в химической связи Те-О участвует только две из них, при этом неподеленная пара на атоме Те $s$-типа находится в несвязанном состоянии и локализована на атоме теллура. 


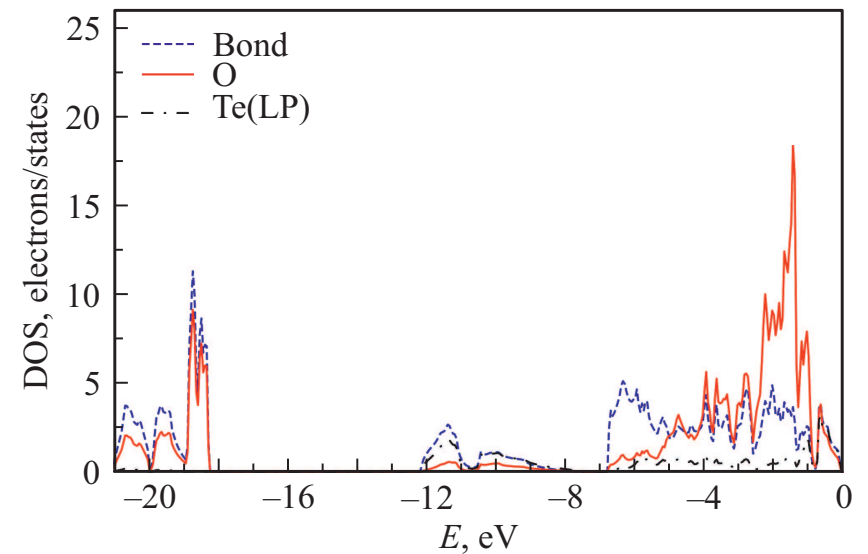

Рис. 5. Спроектированная плотность электронных состояний кристалла $\gamma-\mathrm{TeO}_{2}$ для орбиталей локализованных на связи $\mathrm{Te}-\mathrm{O}$ (штриховая линия), для орбиталей неподеленных пар атома О (сплошная линия) и для орбиталей неподеленной пары атома Те (штрихпунктирная линия).

Чтобы установить вклад каждого типа орбиталей в электронную структуру, на рис. 5 построена спроектированная плотность электронных состояний.

Как видно из зонной структуры (рис. 3) можно выделить три валентных зоны, а именно нижняя зона в области $[-21:-18] \mathrm{eV}$, средняя зона $[-12:-8] \mathrm{eV}$ и верхняя $[-6.5: 0] \mathrm{eV}$. Глубинная валентная зона в области $[-40:-35] \mathrm{eV}$ это сильно локализованные остовные состояния на $4 d$-орбиталях атома Те и в химической связи участия не принимают. Все зоны разделены между собой запрещенными зонами в которых электронные состояния полностью отсутствуют. Нижняя зона отделена от средней запрещенной зоной величина которой составляет порядка $6 \mathrm{eV}$ и состоит, как видно из рис. 5, из состояний, образованных орбиталями атома кислорода как локализованных, так и не локализованных на связях Те-О. Средняя зона состоит из четырех ветвей, которые имеют максимальную дисперсию по ЗБ, и отделена от верхней зоны величина которой небольшая и составляет всего $1 \mathrm{eV}$. В формировании этой зоны участвуют, главным образом, орбитали атомов О, локализованных на связи, и орбитали неподеленной пары атомов Те. Самая верхняя валентная зона сформирована всеми тремя типами орбиталей, причем вклад орбиталей, локализованных на связях, доминирует в низкоэнергетичной области этой зоны, а в высокоэнергетичной области основной вклад в состояния дают орбитали неподеленной пары атомов О и Те. При этом большую дисперсию ветвей средней валентной зоны можно объяснить высокой степенью делокализации орбиталей неподеленной пары атомов Те, которые, как отмечалось выше, вносят существенный вклад в формирование этих состояний. Таким образом, из всех орбиталей атома теллура заполнена только одна $5 s$-орбиталь и следовательно валентность этого атома равна четырем.

\section{5. Расчеты нелинейных оптических свойств $\mathrm{TeO}_{2}$}

Расчет нелинейной диэлектрической восприимчивости был выполнен путем разложения в ряд зависимости диэлектрической поляризации $\mathbf{P}$ от напряженности электрического поля $\mathbf{E}$ :

$$
\begin{aligned}
\mathscr{P}_{i}=\mathscr{P}_{i}^{S} & +\varepsilon_{0}\left(\sum_{j=0}^{3} \chi_{i j}^{(1)} \mathscr{E}_{j}+\sum_{j, k=0}^{3} \chi_{i j k}^{(2) \mathscr{E}_{j} \mathscr{E}_{k}}\right. \\
& \left.+\sum_{j, k, l=0}^{3} \chi_{i j k l}^{(3)} \mathscr{E}_{j} \mathscr{E}_{k} \mathscr{E}_{l}\right)+\ldots,
\end{aligned}
$$

где $\chi_{i j}^{(1)}$ - элемент тензора линейной восприимчивости, a $\chi_{i j k}^{(2)}$ и $\chi_{i j k l}^{(3)}$ - элементы тензора квадратичной и кубической нелинейной восприимчивости, соответственно.

Величина диэлектрической поляризации периодической бесконечной системы не может быть определена однозначно в прямом пространстве как сумма дипольных моментов частиц ввиду произвольности в выборе единицы объема, в котором ведется суммирование, поэтому расчет ведется в обратном пространстве с помощью фазы Берри [14]. Следует отметить, что формула (2) учитывает только поляризацию, связанную с перераспределением электронной плотности, т.е. атомы остаются неподвижными (статический предел). Для данного случая тензор квадратичной восприимчивости является симметричным относительно перестановок индексов (симметрия Клейнмана).

Пространственная симметрия вещества накладывает ограничения на число ненулевых компонент тензора третьего ранга. Согласно симметрийному анализу [23] в случае $\alpha-\mathrm{TeO}_{2}$ всего шесть ненулевых элемента причем для них справедливо следующее:

$$
\chi_{x y z}^{(2)}=-\chi_{y x z}^{(2)}, \quad \chi_{x z y}^{(2)}=-\chi_{y z x}^{(2)}, \quad \chi_{z x y}^{(2)}=-\chi_{z y x}^{(2)} .
$$

Однако согласно симметрии Клейнмана также справедливо и такие равенства

$$
\chi_{x y z}^{(2)}=\chi_{y x z}^{(2)}, \quad \chi_{x z y}^{(2)}=\chi_{y z x}^{(2)}, \quad \chi_{z x y}^{(2)}=\chi_{z y x}^{(2)} .
$$

Очевидно, что одновременное выполнение равенств (3) и (4) возможно только в случае отсутствия нелинейного отклика второго порядка, т.е. все компоненты квадратичного тензора нелинейной восприимчивости равны нулю.

В случае $\gamma-\mathrm{TeO}_{2}$ точечная симметрия которого (точечная группа 222) определяет число ненулевых компонент нелинейных восприимчивости. Для квадратичной восприимчивости таких компонент шесть, а именно $\chi_{x y z}^{(2)}, \chi_{x z y}^{(2)}, \chi_{y z x}^{(2)}, \chi_{y x z}^{(2)}, \chi_{z x y}^{(2)}$ и $\chi_{z y x}^{(2)}$ [23]. Согласно симметрии Клейнмана (симметрия тензора квадратичной восприимчивости относительно перестановки индексов все компоненты равны. Для кубической нелинейной 
восприимчивости имеется три ненулевых диагональных элемента $\chi_{x x x x}^{(3)}, \chi_{y y y y}^{(3)}, \chi_{z z z z}^{(3)}$ и 18 элементов с индексами эквивалентными в парах, и в статическом пределе это компоненты $\chi_{x x y y}^{(3)}, \chi_{x x z z}^{(3)}$ и $\chi_{y y z z}^{(3)}$.

Если в (2) положить $i=z, j=x, k=y$, то учитывая симметрию кристалла, которое накладывает ограничение на число ненулевых компонент, зависимость поляризации от приложенного поля будет выглядеть следующим образом:

$$
\mathscr{P}_{z}=\varepsilon_{0}\left(\chi_{z x y}^{(2)} \mathscr{E}_{x} \mathscr{E}_{y}+\chi_{z y x}^{(2)} \mathscr{E}_{y} \mathscr{E}_{x}\right)=2 \varepsilon_{0} \chi_{z x y}^{(2)} \mathscr{E}_{x} \mathscr{E}_{y}
$$

ввиду того, что в статическом пределе, как упоминалось выше, тензор нелинейной восприимчивости симметричен относительно перестановок индексов и тензор нелинейной кубической восприимчивости имеет только диагональные и эквивалентные в парах индексов ненулевые компоненты.

Была рассчитана зависимость поляризации от напряженности электрического поля, приложенного одновременно в направлениях $x$ и $y$, а именно получен набор точек $P_{z}^{i}\left(E_{i}\right)$, которые аппроксимировались квадратным многочленом и таким образом были получено значение нелинейной восприимчивости равное $\chi_{x y z}^{(2)}=1.86 \mathrm{pm} / \mathrm{V}$. Полученное значение можно сравнить с величиной квадратичной нелинейной восприимчивости такого эталонного материала как кварц $\alpha-\mathrm{SiO}_{2}$, которое равно $\chi_{x y z}^{(2)}=0.6 \mathrm{pm} / \mathrm{V}$ [23]. Следовательно величина квадратичной нелинейной восприимчивости $\gamma-\mathrm{TeO}_{2}$ в несколько раз больше чем в кварце. Таким образом можно сделать вывод, что несмотря на небольшие структурные изменения $\gamma$-фазы относительно $\alpha$-фазы оксида теллуpa, понижение симметрии позволяет реализовать свой потенциал в нелинейной оптике в качестве активного элемента генерации второй гармоники.

\section{6. Заключение}

В работе представлены результаты комплексного изучения структурных свойств с применением теории функционала плотности с учетом хаббардовских поправок. Данные результаты показали хорошее согласие с экспериментом. Путем представления $\alpha-\mathrm{TeO}_{2}$ в группе низкосимметричной ромбической фазы выявлена в целом схожесть структур, а отличительная особенность заключается в сдвиге атомов кислорода $\mathrm{O}_{2}$ вдоль оси $C_{4}$. Выполнены расчеты электронной структуры $\gamma-\mathrm{TeO}_{2}$ с помощью квазичастичного метода $G_{0} W_{0}$, в результате получена оценка величины запрещенной зоны и установлено, что $\gamma-\mathrm{TeO}_{2}$ широкозонный полупроводник с непрямым переходом. Были построены максимально локализованные функции Ванье для этого кристалла, что в совокупности с анализом вклада каждой орбитали в плотность электронных состояний позволило определить тип химической связи в $\gamma-\mathrm{TeO}_{2}$. Наконец, с помощью метода фаз Берри была рассчитана зависимость вектора поляризации от электрического поля, аппроксимация которой квадратным многочленом позволила вычислить значение квадратичной нелинейной восприимчивости, которое в несколько раз превосходит аналогичное значение для кварца. Полученные результаты позволяют лучше понять фундаментальные свойства целого семейства простых и сложных оксидов теллура. Авторы надеются пробудить интерес к экспериментальному изучению нелинейных оптических свойств гамма фазы оксида теллура.

\section{Благодарности}

Представленные в настоящей работе расчеты были частично выполнены, в Ресурсном Центре „Вычислительный Центр“ СПбГУ, и с использованием вычислительных ресурсов суперкомпьютерного центра СанктПетербургского политехнического университета Петра Великого (www.scc.spbstu.ru).

\section{Финансирование работы}

Работа была выполнена при финансовой поддержке Российского фонда фундаментальных исследований (номер гранта 18-03-00750).

\section{Конфликт интересов}

Авторы заявляют, что у них нет конфликта интересов.

\section{Список литературы}

[1] S.-H. Kim, T. Yoko, S. Sakka. J. Am. Ceram. Soc. 76, 2486 (1993).

[2] V. Voloshinov, V. Molchanov. Opt. Laser Technol. 27, 307 (1995).

[3] A. Mirgorodsky, T. Merle-Mèjean, J.-C. Champarnaud, P. Thomas, B. Frit. J. Phys. Chem. Solids 61, 501 (2000).

[4] A.D. Becke. J. Chem. Phys. 140, 18A301 (2014).

[5] A. Marini, P. García-González, A. Rubio. Phys. Rev. Lett. 96, 136404 (2006).

[6] M. Shishkin, G. Kresse. Phys. Rev. B 75, 235102 (2007).

[7] X. Gonze, B. Amadon, P.-M. Anglade, J.-M. Beuken, F. Bottin, P. Boulanger, F. Bruneval, D. Caliste, R. Caracas, M. Côtè, T. Deutsch, L. Genovese, P. Ghosez, M. Giantomassi, S. Goedecker, D. Hamann, P. Hermet, F. Jollet, G. Jomard, S. Leroux, M. Mancini, S. Mazevet, M. Oliveira, G. Onida, Y. Pouillon, T. Rangel, G.-M. Rignanese, D. Sangalli, R. Shaltaf, M. Torrent, M. Verstraete, G. Zerah, J. Zwanziger. Comput. Phys. Commun. 180, 2582 (2009).

[8] X. Gonze, G. Rignanese, M. Verstraete, J. Beuken, Y. Pouillon, R. Caracas, F. Jollet, M. Torrent, G. Zerah, M. Mikami, P. Ghosez, M. Veithen, J.-Y. Raty, V. Olevano, F. Bruneval, L. Reining, R. Godby, G. Onida, D. Hamann, D. Allan. Zeitschrift für Kristallographie. Cryst. Mater. 220, 558 (2005).

[9] J.P. Perdew, Y. Wang. Phys. Rev. B 45, 13244 (1992).

[10] V.I. Anisimov, J. Zaanen, O.K. Andersen. Phys. Rev. B 44, 943 (1991). 
[11] E.M. Roginskii, V.G. Kuznetsov, M.B. Smirnov, O. Noguera, J.-R. Duclère, M. Colas, O. Masson, P. Thomas. J. Phys Chem C 121, 12365 (2017).

[12] D.R. Hamann. Phys. Rev. B 88, 085117 (2013).

[13] H.J. Monkhorst, J.D. Pack. Phys. Rev. B 13, 5188 (1976).

[14] D. Vanderbilt, R.D. King-Smith. Phys. Rev. B 48, 4442 (1993).

[15] G. Onida, L. Reining, A. Rubio. Rev. Mod. Phys. 74, 601 (2002).

[16] Yu.E. Kitaev, A.G. Panfilov, V.P. Smirnov, P. Tronc. Phys. Rev. E 67, 011907 (2003).

[17] H.T. Stokes, D.M. Hatch. Phys. Rev. B 65, 144114 (2002). 14.

[18] G. Vrillet, C. Lasbrugnas, P. Thomas, O. Masson, V. Couderc, A. Barthélémy, J.-C. Champarnaud-Mesjard. J. Phys. C: 21, 4611 (1988).

[19] A. Mirgorodsky, T. Merle-Méjean, J.-C. Champarnaud, P. Thomas, B. Frit. J. Phys. Chem. Solids 61, 501 (2000).

[20] N. Berkaïne, E. Orhan, O. Masson, P. Thomas, J. Junquera. Phys. Rev. B. 83, 245205 (2011).

[21] A.A. Mostofi, J.R. Yates, G. Pizzi, Y.-S. Lee, I. Souza, D. Vanderbilt, N. Marzari. Comput. Phys. Commun. 185, 2309 (2014).

[22] Y. Hinuma, G. Pizzi, Y. Kumagai, F. Oba, I. Tanaka. Comput. Mater. Sci. 128, 140 (2017).

[23] R.W. Boyd. Nonlinear Optics. Academic Press Inc (2007), N.Y. 613 p.

Редактор Ю.Э. Китаев 